\title{
Signs of new physics in top quark pair production associated with a neutrino pair at the $\mathrm{LHC}$
}

\author{
Daruosh Haji Raissi $\odot,{ }^{1}$ Javad Ebadi $\odot,{ }^{2}$ and Mojtaba Mohammadi Najafabadi $\oplus^{3}$ \\ ${ }^{1}$ Faculty of Sciences, Department of Physics, Ayatollah Amoli Branch, \\ Islamic Azad University, Amol 678, Mazandaran, Iran \\ ${ }^{2}$ School of Physics, Institute for Research in Fundamental Sciences (IPM) \\ P.O. Box 19395-5531, Tehran, Iran \\ ${ }^{3}$ School of Particles and Accelerators, Institute for Research in Fundamental Sciences (IPM) \\ P.O. Box 19395-5531, Tehran, Iran
}

(Received 30 October 2019; accepted 9 March 2020; published 5 May 2020)

\begin{abstract}
In this paper, we examine the interactions of top quarks with the $Z$ boson using the top quark pair production associated with the neutrino pair $\left(t \bar{t} \nu_{l} \bar{\nu}_{l}\right)$ at the LHC. In particular, potential constraints on the anomalous electroweak top quark interactions are determined by considering two opposite-sign charged leptons, missing energy, and two b-tagged jets in the final state. The analysis is performed for a high luminosity scenario of the LHC with an integrated luminosity of $3 \mathrm{ab}^{-1}$ of proton-proton collisions at a center-of-mass energy of $14 \mathrm{TeV}$. The $95 \%$ confidence intervals are computed on the anomalous couplings considering a realistic detector simulation of an upgraded CMS detector including an average of 200 proton-proton interactions per bunch crossing. We find that the $t \bar{t} \nu_{l} \bar{\nu}_{l}$ channel can provide stringent bounds on the relevant Wilson coefficients and has the potential to serve as an additional handle beside the $t \bar{t} Z\left(Z \rightarrow l^{+} l^{-}\right)$and other channels to search for new physics.
\end{abstract}

DOI: 10.1103/PhysRevD.101.095002

\section{INTRODUCTION}

The main task of the Large Haron Collider (LHC) is to make an inquiry for possible effects of new physics beyond the Standard Model (SM). As the collected data by the LHC experiments have increased, the motivated models beyond the SM are being studied in detail and are strongly constrained. As a result, the phenomenological studies have become largely model independent, and data are interpreted in the framework of the SM effective field theory (EFT) [1-3]. The effective field theory extension of the SM has become a popular theoretical framework to look for beyond the SM effects and has received a lot of attention during the last years [4-46].

The effective field theory extension of SM is a power tool which could be considered as a bridge between the measurements at a low energy scale and the unknown UV completion theory. The LHC experiments could observe the impacts of non-SM physics provided that its energy scale would be below the energy of the related hard processes. Otherwise, the new physics effects should be

Published by the American Physical Society under the terms of the Creative Commons Attribution 4.0 International license. Further distribution of this work must maintain attribution to the author(s) and the published article's title, journal citation, and DOI. Funded by SCOAP ${ }^{3}$. probed through the precise measurements of the interactions of the SM particles. As all the measurements have been found to be consistent with the SM predictions, one expects that the possible heavy degrees of freedom are apart from the SM content in mass. Within the framework of the effective field theory of the SM, the new physics effects can be parametrized by a series of $\mathrm{SU}(3)_{\mathrm{c}} \times \mathrm{SU}(2)_{\mathrm{L}} \times \mathrm{U}(1)_{\mathrm{Y}}$ gauge invariant dimension-six operators $\mathcal{O}_{i}$ built out of the SM fields. The coefficients of the operators are suppressed by the inverse power of the new physics characteristic scale $\Lambda[2,3]$,

$$
\mathcal{L}_{\text {eff }}=\mathcal{L}_{\mathrm{SM}}+\sum_{i} \frac{C_{i}^{(6)} O_{i}^{(6)}}{\Lambda^{2}}
$$

where $\mathcal{L}_{\mathrm{SM}}$ is the known SM Lagrangian and $C_{i}^{(6)}$ 's are the so-called Wilson coefficients, which are dimensionless. The leading contributions arise from the operators of dimension-six, and the Wilson coefficients are considered as a priori free parameters when we constrain a generic model beyond the SM. A list of dimension-six operators $O_{i}^{(6)}$ could be found in Refs. [2,3]. The validity of the effective field theory extension of the SM has been investigated in Ref. [47], where it has been shown that the validity range of EFT could not be derived only on the basis of low energy information and the conditions for an 
EFT to provide an appropriate low-energy description of an underlying model beyond the SM are discussed.

In the present work, we perform a search for beyond the SM effects in the context of the SM effective field theory (SMEFT) through the production of $t \bar{t}$ in association with a neutrino pair at the LHC. The Wilson coefficients of the relevant dimension-six operators are constrained. There are 59 operators of dimension-six that form the so-called Warsaw basis [3], among them the four most relevant linear combinations, as represented in Ref. [9], are selected. The study is performed for a high luminosity scenario of the LHC at a center-of-mass energy of $14 \mathrm{TeV}$ using an integrated luminosity of $3 \mathrm{ab}^{-1}$. Constraints at $95 \%$ confidence level are obtained on the relevant Wilson coefficients using the dilepton channel of the top pair events considering an upgraded CMS detector [48] and an average of 200 proton-proton interactions in each bunch crossing.

The production cross section of $t \bar{t} Z\left(Z \rightarrow l^{+} l^{-}\right)$has been measured by the ATLAS and CMS Collaborations using proton-proton collisions at $\sqrt{s}=13 \mathrm{TeV}$, and constraints have been applied on the Wilson coefficients $[49,50]$. The expected sensitivity of the CMS experiment for the anomalous electroweak top quark interactions has been provided for a HL-LHC scenario with $3 \mathrm{ab}^{-1}$ at a center-ofmass energy of $14 \mathrm{TeV}$ in Ref. [51]. The constraints have been obtained based on the measurements of the differential cross section of the $t \bar{t} Z$ process in the three lepton final state.

This article is organized as follows. In Sec. II, the theoretical framework and the contributing dimension-six operators, which affect $t \bar{t} \nu_{l} \bar{\nu}_{l}$, are discussed in short. Section III presents the production of $t \bar{t} \nu_{l} \bar{\nu}_{l}$ process. The present constraints on the electroweak anomalous top- $Z$ interactions are given in Sec. III. In Sec. IV, we discuss the event generation, detector simulation, and the analysis strategy. The estimated sensitivity that could be achieved from the HL-LHC are presented in Sec. V. A summary and conclusions are given in Sec. VI.

\section{EFFECTIVE LAGRANGIAN}

As it was mentioned, in the case that possible new particles are too heavy with respect to the LHC energy scale and are not produceable on shell, one can use a low energy effective theory to describe the observables and look for possible new physics effects. In this section, the effective Lagrangian up to dimension-six operators, which modify the top quark and $Z$ boson interactions, is introduced. The anomalous interactions between the top quark and gluons are not considered here as they have been strongly constrained using the $t \bar{t}+$ jets process [52]. We also neglect the anomalous $W t b$ coupling in the current analysis due to the tight bounds obtained by a single top quark production and $W$-polarization measurements [53]. The most general effective Lagrangian describing the $t \bar{t} Z$ interaction can be written as $[54,55]$,

$$
\begin{aligned}
\mathcal{L}_{Z \bar{t} \bar{t}}= & e \bar{u}_{t}\left[\left(C_{1, V}+\gamma^{5} C_{1, A}\right) \gamma^{\mu}+\frac{i \sigma^{\mu \nu} q_{\nu}}{m_{Z}}\left(C_{2, V}+i \gamma^{5} C_{2, A}\right)\right] \\
& \times v_{\bar{t}} Z_{\mu},
\end{aligned}
$$

where $\sigma^{\mu \nu}=\frac{i}{2}\left[\gamma^{\mu}, \gamma^{\nu}\right]$ and $q=p_{t}-p_{\bar{t}}$. Within the SM at tree level, the vector and axial couplings are

$$
\begin{gathered}
C_{1, V}=C_{V}^{\mathrm{SM}}=\frac{-2 Q_{t} \sin ^{2} \theta_{W}+T_{t}^{3}}{2 \sin \theta_{W} \cos \theta_{W}}, \\
C_{1, A}=C_{A}^{\mathrm{SM}}=-\frac{T_{t}^{3}}{2 \sin \theta_{W} \cos \theta_{W}},
\end{gathered}
$$

where $\theta_{W}$ is the weak mixing angle, $Q_{t}$ is the top quark electric charge which is equal to $2 / 3$, and $T_{t}^{3}=1 / 2$. The values of $C_{V}^{\mathrm{SM}}$ and $C_{A}^{\mathrm{SM}}$ in the $\mathrm{SM}$ are 0.244 and -0.601 , respectively. In the SM, at tree level, $C_{2, V}$ and $C_{2, A}$ are zero; however, $C_{2, V}$ receives corrections of the order of $10^{-4}$ from one-loop diagrams, and $C_{2, A}$ gets corrections from three-loop diagrams [56-58]. Following the parametrization of Ref. [9], the relevant Wilson coefficients are $c_{t Z}, c_{t Z}^{[I]}$, $c_{\phi t}$, and $c_{\phi Q}^{-}$. These coefficients have a simple translation to the Wilson coefficients in the Warsaw basis [3], which can be found in the following [9]:

$$
\begin{aligned}
c_{t Z} & =\operatorname{Re}\left(-\sin \theta_{W} C_{u B}^{(33)}+\cos \theta_{W} C_{u W}^{(33)}\right), \\
c_{t Z}^{[I]} & =\operatorname{Im}\left(-\sin \theta_{W} C_{u B}^{(33)}+\cos \theta_{W} C_{u W}^{(33)}\right), \\
c_{\phi t} & =C_{\phi t}=C_{\phi u}^{(33)}, \\
c_{\phi Q}^{-} & =C_{\phi Q}=C_{\phi q}^{1(33)}-C_{\phi q}^{3(33)} .
\end{aligned}
$$

Similar to the recent CMS experiment analyses [49,51], we consider $c_{t Z}, c_{t Z}^{[I]}, c_{\phi t}$, and $c_{\phi Q}^{-}$in this work and set other Wilson coefficients to zero. Setting $C_{\phi q}^{3(33)}$ and $C_{u W}^{(33)}$ to zero guarantees the $W t b$ vertex is consistent with the SM.

\section{PRODUCTION OF $\bar{t}_{\nu_{l}} \overline{\nu_{l}}$ AT THE LHC}

In this section, the production of $t \bar{t} \nu_{l} \bar{\nu}_{l}$ in proton-proton collisions at the LHC is discussed. Within the SM framework, at leading order, the production of $t \bar{t} \nu_{l} \bar{\nu}_{l}$ proceeds via gluon-gluon fusion and quark-antiquark annihilation in both the $s$ and t channel, where the pair of neutrino comes from the $Z$ boson decay. Figure 1 shows the representative Feynman diagrams at leading order at the LHC. At $\sqrt{s}=14 \mathrm{TeV}$, the leading order cross section of the $t \bar{t} \nu_{l} \bar{\nu}_{l}$ process is $143 \mathrm{fb}$ from which around $72 \%$ comes from the gluon-gluon fusion. The next to leading order (NLO) QCD cross section, obtained with MadGraph5_aMC@NLO [59,60] is $195 \mathrm{fb}$.

The new physics Lagrangian introduced in Eq. (2) affects the $t \bar{t} Z$ vertex in the $t \bar{t} Z$ production in proton-proton collisions. The impacts of the anomalous couplings on the total cross section and the differential distributions of 


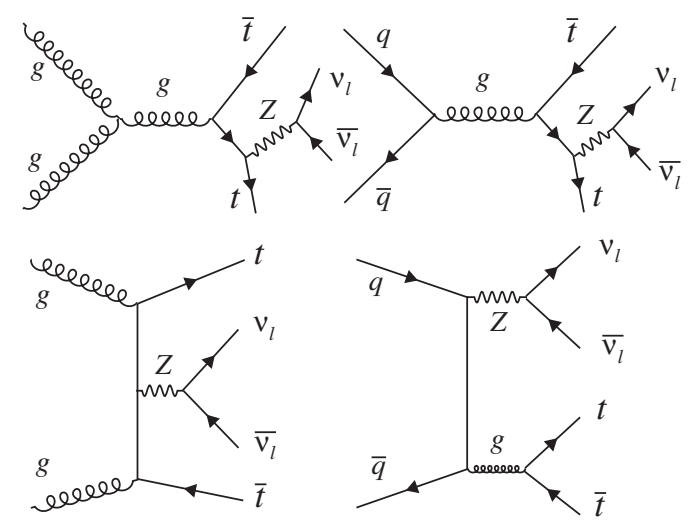

FIG. 1. Representative Feynman diagrams for $t \bar{t} \nu_{l} \bar{\nu}_{l}$ production at leading order in proton-proton collisions the LHC.

$t \bar{t} Z$ production have been extensively studied in Refs. $[23,55]$. According to these studies, in the presence of the defined Wilson coefficients in Eq. (2), the production rate receives remarkable modification with respect to the SM case. In addition, the kinematic distributions of the final state particles are strongly affected by the anomalous couplings. Particularly, the electroweak dipole couplings $C_{2, V}$ and $C_{2, A}$ are expected to lead an enhancement in the tail of the momentum distributions of the final particles. This is because of the Lorentz structures of these couplings in the $t \bar{t} Z$ vertex, which contains the $Z$ boson momentum. As a result, in this study where the $Z$ boson in $t \bar{t} Z$ production decays to a pair of neutrino, we expect an enhancement in the tail of missing transverse energy $\left(E_{\mathrm{T}}^{\text {miss }}\right)$ distribution. Therefore, in the next sections, we focus on the $E_{\mathrm{T}}^{\text {miss }}$ distribution to constrain the Wilson coefficient.

The observed sensitivity of the anomalous electroweak top quark interactions have been determined based on the measurements of the differential cross section of the $t \bar{t} Z$ process in the three lepton final state. The limits at 95\% C.L. are [49]

$$
\begin{aligned}
-1.1 \leq c_{t Z} \leq 1.1, & -1.2 \leq c_{t Z}^{[I]} \leq 1.2, \\
0.3 \leq c_{\phi t} \leq 5.4, & -4.0 \leq c_{\phi Q}^{-} \leq 0.0 .
\end{aligned}
$$

These bounds have been obtained using $77.5 \mathrm{fb}^{-1}$ of the LHC data at a center-of-mass energy of $13 \mathrm{TeV}$. Expected 95\% C.L. limits for a HL-LHC scenario with $3 \mathrm{ab}^{-1}$ at a center-of-mass energy of $14 \mathrm{TeV}$ are [51]

$$
\begin{array}{ll}
-0.52 \leq c_{t Z} \leq 0.51, & -0.54 \leq c_{t z}^{[I]} \leq 0.51, \\
-0.89 \leq c_{\phi t} \leq 0.89, & -0.75 \leq c_{\phi Q}^{-} \leq 0.73 .
\end{array}
$$

These constraints have been derived for an upgraded CMS detector with the same analysis strategy followed in Ref. [49].

In this work, the calculations for the cross sections are performed at leading-order using MadGraph5_aMC@NLO package in the context of SMEFT following the

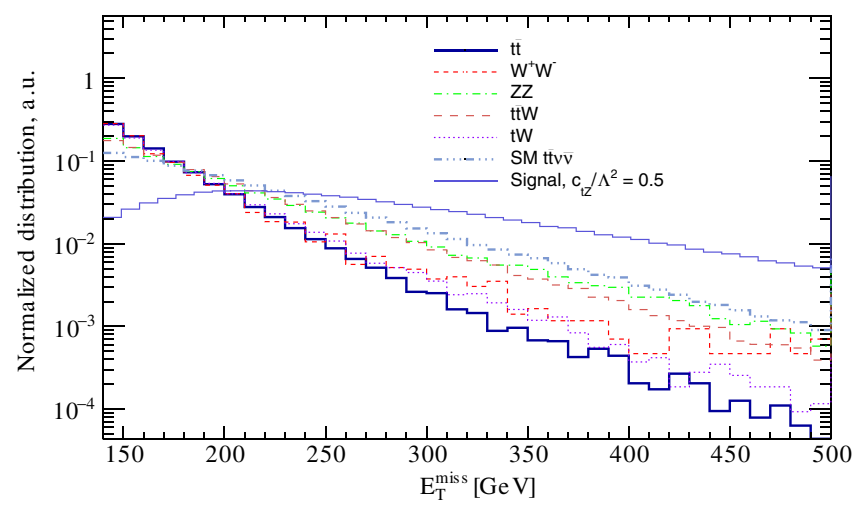

FIG. 2. Plot shows the normalized distribution of the missing transverse momentum for the signal scenario with $c_{t Z} / \Lambda^{2}=$ $0.5 \mathrm{TeV}^{-2}$ and for some of the background processes such $t \bar{t}, t \bar{t} W, t W$, diboson, and SM production of $t \bar{t} \nu_{l} \bar{\nu}_{l}$.

parametrization adopted in Ref. [9]. The model implementation has been performed with the FeynRules package [61] for generation of the related UFO file model that is inserted into the MadGraph5_aMC@NLO. ${ }^{1}$ The details of simulations, analysis strategy, and determination of the constraints on the Wilson coefficients are discussed in the next sections.

\section{SIMULATION AND ANALYSIS STRATEGY}

In this section, the details of simulation and the analysis strategy for probing the effective SM in the $t \bar{t}$ production associated with a pair of neutrino are described. In order to have a clean signature, we consider the dileptonic decay of the $t \bar{t}$. Consequently, the final state consists of two isolated charged leptons (electron and/or muon), two jets originating from the hadronization of bottom quarks, and large missing transverse energy. The major background processes which are included in this analysis are $t \bar{t}, t \bar{t} Z\left(\rightarrow \nu_{l} \bar{\nu}_{l}\right)$, single top $t W$-channel, $t \bar{t} W^{ \pm}, t \bar{t} H, W^{ \pm} W^{ \pm}, Z Z$, and $W^{ \pm} Z$.

The generation of signal and background events are done with MadGraph5_aMC@NLO. Then, the events are passed through PYTHIA $[62,63]$ to perform parton showering, hadronization, and decays of unstable particles. The events are generated at $\sqrt{s}=14 \mathrm{TeV}$ at the LHC with the NNPDF2.3 as the proton parton distribution functions [64]. The SM input parameters for generation of the events are $m_{t}=173.3 \mathrm{GeV}$ and $m_{Z}=91.187 \mathrm{GeV}$, $m_{W}=80.385 \mathrm{GeV}, m_{H}=125.0 \mathrm{GeV}$. Before we perform an analysis with a realistic detector simulation, it is worth presenting the distribution of the missing transverse momentum which is one the main characteristic of the signal process. Missing transverse momentum distribution $\left(\left|\sum_{i}\left(\vec{p}_{\nu^{i}, T}+\vec{p}_{\bar{\nu}^{i}, T}\right)\right|\right)$ is depicted in Fig. 2. The signal distribution is presented for the case of $c_{t Z} / \Lambda^{2}=$ $0.5 \mathrm{TeV}^{-2}$, and for comparison, the distributions for the

\footnotetext{
${ }^{1}$ The UFO file of the model has been taken from https:// feynrules.irmp.ucl.ac.be/wiki/dim6top.
} 
major backgrounds like $t \bar{t}, t \bar{t} W, t W, Z Z, W^{+} W^{-}$and SM production of $t \bar{t}_{l} \bar{\nu}_{l}$ are shown. It can be seen that the tail of the missing transverse momentum distribution is highly sensitive to the signal so that most of the backgrounds are peaked towards low missing transverse momentum. Therefore, in this work to perform the search and study the sensitivity, we concentrate on the tail of the missing transverse momentum distribution.

The detector response simulation is done using DELPHES [65] package for an upgraded CMS detector [66]. The events are simulated by taking into account the additional proton-proton interactions for each bunch crossing (pileup) with a mean number of pileup interactions of 200. The jet finding process is performed using FastJet package [67] and the anti- $k_{t}$ algorithm [68] is utilized for reconstruction of jets with a distance parameter of 0.4 considering a pileup correction. The b-quark jet tagging efficiency and the rates of misidentification are dependent on the jets transverse momentum and have the following parametrizations [69]:

Light-flavor jets: $0.01+0.000038 \times p_{\mathrm{T}}$,

Misidentification rate of the c-jet: $0.25 \times \tanh \left(0.018 \times p_{\mathrm{T}}\right) \times \frac{1.0}{1+0.0013 \times p_{\mathrm{T}}}$,

b-tagging efficiency: $0.85 \times \tanh \left(0.0025 \times p_{\mathrm{T}}\right) \times \frac{25.0}{1+0.063 \times p_{\mathrm{T}}}$,

where the transverse momentum $p_{\mathrm{T}}$ is in $\mathrm{GeV}$ unit. The efficiency of $\mathrm{b}$ tagging for a jet with transverse momentum of $30 \mathrm{GeV}$ is around $55 \%$ and the $\mathrm{c}$ jet and light flavor jets misidentification rates are $12 \%$ and $1 \%$, respectively.

In order to select signal events, it is required to have two opposite sign charged leptons with transverse momenta $p_{\mathrm{T}}$ and pseudorapidity $\eta_{l}$ satisfying $p_{\mathrm{T}}>20 \mathrm{GeV}$ and $\left|\eta_{l}\right|<3$.0. This requirement fulfills the high level trigger (HLT) condition [70]. The accepted charged leptons (muon and/or electron) are required to have a relative isolation $I_{\text {Rel }}<0.15$, where $I_{\text {Rel }}$ is defined as the scalar sum of transverse momenta of all particles inside a cone of size 0.4 around the lepton direction, except the lepton, divided by the $p_{\mathrm{T}}$ of lepton. Events are demanded to have exactly two b jets with $p_{\mathrm{T}}>30 \mathrm{GeV}$ and $|\eta|<4.0$. To make sure all objects are well-isolated, the angular separation between the leptons and jets are required to satisfy $\Delta R\left(l^{ \pm}, b\right.$-jet $)>0.4$, where $\Delta R=\sqrt{(\Delta \eta)^{2}+(\Delta \phi)^{2}}$. In order to reduce the SM background contributions, an additional cut is applied on the missing transverse energy so that the signal-to-background ratio is good enough to achieve the best sensitivity. The efficiencies of the cuts for the signal scenario $c_{t Z} / \Lambda^{2}=0.5 \mathrm{TeV}^{-2}$ and the main background processes are presented in the Table I. In particular, the efficiencies are given for illustration in a region of missing transverse energy above $E_{\mathrm{T}}^{\text {miss }} \geq 400 \mathrm{GeV}$.
The contributions of background processes, such as $Z Z$, $W^{ \pm} W^{\mp}$, and $W^{ \pm} Z, t \bar{t} H$, are found to be negligible in this region. As the contribution of background processes overwhelms the signal at low values of cut on the magnitude of missing transverse energy, the concentration is on a region where the ratio of signal-to-background is large enough to find the exclusion limits. Because the signal events tend to have larger $E_{T}^{\text {miss }}$ values with respect to the background, the $E_{\mathrm{T}}^{\text {miss }}$ region above $200 \mathrm{GeV}$ will be chosen to obtain the limits.

The enhancement of the cross section in the presence of the anomalous couplings leads violation of the unitarity at very high energies. One needs to ensure the validity of the SM effective theory in this analysis. There are studies where the authors discussed the validity of effective theory, which, for instance, could be found in Refs. [9,47,71]. In the present study, an upper bound of $E_{T}^{\text {miss }}<1.5 \mathrm{TeV}$ is applied to avoid unitarity violation.

\section{RESULTS}

This section is dedicated to presenting the potential sensitivity of the top pair production in association with a pair of neutrinos to the Wilson coefficients. The results are presented for the collisions at a center-of-mass energy of $14 \mathrm{TeV}$ and are corresponding to an integrated luminosity of $3 \mathrm{ab}^{-1}$.

TABLE I. Expected efficiencies after cuts for signal scenario $c_{t Z} / \Lambda^{2}=0.5 \mathrm{TeV}^{-2}$ and the main SM background processes. Detailed description of the cuts are presented in the text.

\begin{tabular}{lcccc}
\hline \hline Cut & $c_{t Z} / \Lambda^{2}=0.5 \mathrm{TeV}^{-2}$ & $\mathrm{SM} t \bar{t} \nu_{l} \bar{\nu}_{l}$ & $t W$ & $t \bar{t}$ \\
\hline $2 l^{ \pm}$, jets, and $b$ tagging & 0.19 & 0.18 & 0.05 & 0.17 \\
$E_{\mathrm{T}}^{\text {miss }} \geq 400 \mathrm{GeV}$ & 0.03 & $2 \times 10^{-3}$ & $5.0 \times 10^{-6}$ & $9.2 \times 10^{-6}$ \\
\hline \hline
\end{tabular}


TABLE II. The expected sensitivities on dimension six operator coefficients using $3 \mathrm{ab}^{-1}$ integrated luminosity of data at the LHC with the center-of-mass energy of $14 \mathrm{TeV}$. The $95 \%$ C.L. upper bounds derived from $t \bar{t} Z\left(\rightarrow l^{+} l^{-}\right)$from Ref. [49] with $77.5 \mathrm{fb}^{-1}$ of data, and the projection with $3 \mathrm{ab}^{-1}$ are presented as well. The constraints are given in the unit of $\mathrm{TeV}^{-2}$.

\begin{tabular}{lccc}
\hline \hline Coupling & $\begin{array}{c}\text { Limit from } \\
t \bar{t} Z\left(\nu_{l} \bar{\nu}_{l}\right)\end{array}$ & $\begin{array}{c}\text { Observed limit from } \\
t \bar{t} Z\left(l^{+} l^{-}\right)[49]\end{array}$ & $\begin{array}{c}\text { Projection from } \\
t \bar{t} Z\left(l^{+} l^{-}\right)[51]\end{array}$ \\
\hline$c_{t Z} / \Lambda^{2}$ & {$[-0.74,0.75]$} & {$[-1.1,1.1]$} & {$[-0.52,0.51]$} \\
$c_{t Z}^{[I]} / \Lambda^{2}$ & {$[-0.49,0.49]$} & {$[-1.2,1.2]$} & {$[-0.54,0.51]$} \\
$c_{\phi t} / \Lambda^{2}$ & {$[-0.76,0.67]$} & {$[0.3,5.4]$} & {$[-0.89,0.89]$} \\
$c_{\phi Q}^{-} / \Lambda^{2}$ & {$[-0.44,0.46]$} & {$[-4.0,0.0]$} & {$[-0.75,0.73]$} \\
\hline \hline
\end{tabular}

The Lagrangian introduced in Eq. (2) consists of new momentum dependent tensor structures, which affect the $Z$ boson energy spectrum. Consequently, the missing transverse energy receives considerable impact from the effective $Z t \bar{t}$ couplings. The strategy to derive constraints on the Wilson coefficients is based on the fact that operators

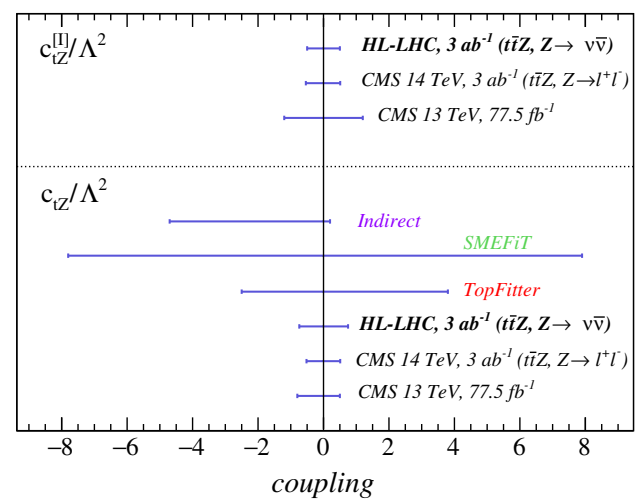

contribute to the tail of missing transverse energy distribution. We consider $E_{\mathrm{T}}^{\mathrm{miss}}$ distribution in three bins of 200-300, 300-400, 400-1500 GeV to set limits where the contributions of SM background are remarkably suppressed. In order to obtain the expected limits at 95\% C.L. on the Wilson coefficients, a binned likelihood function is constructed as a product of Poisson probabilities over three bins of the missing transverse energy. Expected 95\% C.L. intervals from this study for the Wilson coefficients $c_{t Z} / \Lambda^{2}, c_{t Z}^{[I]} / \Lambda^{2}, c_{\phi t} / \Lambda^{2}$, and $c_{\phi Q}^{-} / \Lambda^{2}$ are presented in Table II. The limits have been derived including only statistical uncertainties. Considering detailed systematic uncertainties is beyond the scope of this work and must be performed by the experimental collaborations. The observed $95 \%$ C.L. intervals from the CMS experiment measurement [51] and the expected results from a HL-LHC with $3 \mathrm{ab}^{-1}$ [49] are shown for comparison.

A comparison of the limits from $t \bar{t} \nu_{l} \bar{\nu}_{l}$ and the expected bounds from the projection of the $t \bar{t} Z\left(Z \rightarrow l^{+} l^{-}\right)$rate suggests that $t \bar{t} \nu_{l} \bar{\nu}_{l}$ is an additional channel that can provide the same order sensitivity as $t \bar{t} Z\left(Z \rightarrow l^{+} l^{-}\right)$on the Wilson

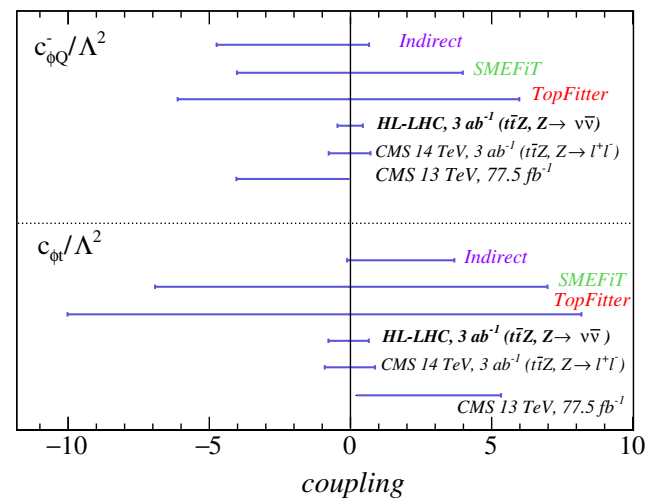

FIG. 3. The expected 95\% C.L. intervals for the Wilson coefficients from this study, the current CMS experiment results based on the $\bar{t} \bar{t} Z\left(Z \rightarrow l^{+} l^{-}\right)$cross section measurement [49], and the CMS projection results at high luminosity. The constraints within the SMEFiT framework [4] and from the TopFitter Collaboration [10] are presented. The indirect bounds from electroweak data at 68\% C.L. are also given [72].
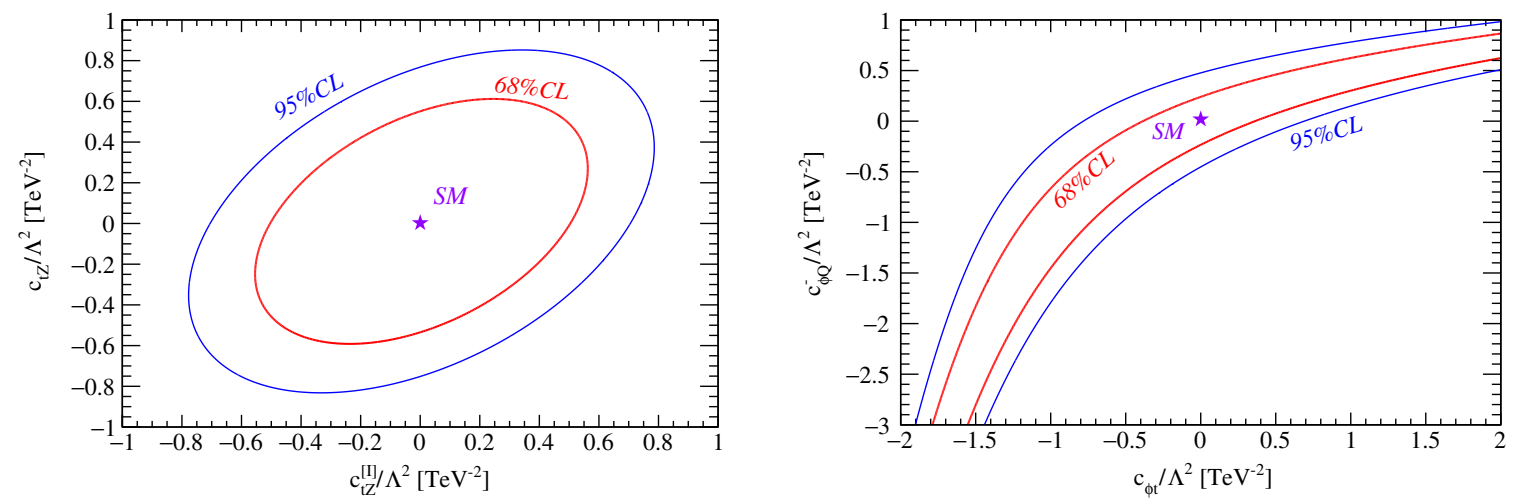

FIG. 4. Two-dimensional scan of the Wilson coefficients in the planes of $\left(c_{t Z} / \Lambda^{2}, c_{t Z}^{[I]} / \Lambda^{2}\right)$ and $\left(c_{\phi t} / \Lambda^{2}, c_{\phi Q}^{-} / \Lambda^{2}\right)$ are depicted. The contours of $68 \%$ and $95 \%$ C.L. are shown in red and blue. The star displays the SM prediction. 
coefficients. Better sensitivity to $c_{\phi t} / \Lambda^{2}$ and $c_{\phi Q}^{-} / \Lambda^{2}$ from this analysis is achievable with respect to the $t \bar{t} Z\left(Z \rightarrow l^{+} l^{-}\right)$ channel.

The expected intervals at 95\% C.L. for the Wilson coefficients from this study, the observed CMS experiment result with $77.5 \mathrm{fb}^{-1}$ from the $t \bar{t} Z$ measurement as well as the CMS experiment projection for a HL-LHC scenario are shown in Fig. 3. The direct constraints from the TopFitter Collaboration and those within the framework of SMEFiT [4] and the indirect bounds at 68\% C.L. from the electroweak precision data [72] are also shown. The SM prediction is shown as a vertical line.

Contours of $68 \%$ (red) and $95 \%$ (blue) C.L. are also obtained for $14 \mathrm{TeV}$ with an integrated luminosity of $3000 \mathrm{fb}^{-1}$. Figure 4 shows the complementary scan of the $c_{t Z} / \Lambda^{2}$ and $c_{t Z}^{[I]} / \Lambda^{2}$ as well as $c_{\phi t} / \Lambda^{2}$ and $c_{\phi Q}^{-} / \Lambda^{2}$ Wilson coefficients in the $2 \mathrm{D}$ plane. The two-dimensional scan shows that correlations are present in the sensitivity of $t \bar{t} \nu_{l} \bar{\nu}_{l}$ to $c_{\phi t}, c_{\phi Q}^{-}$and $c_{t Z}, c_{t Z}^{[I]}$.

\section{SUMMARY AND CONCLUSIONS}

So far, the LHC experiments in runs I and II have found no significant deviation from the SM expectations. In particular, all top quark and Higgs boson properties have been found to be in agreement with the predictions of the SM within the uncertainties. Consequently, for the sake of searching for the effects of possible new physics beyond the SM, one may concentrate on the SM effective field theory framework in which dimension-six operators are considered. The contributions of these operators are suppressed by the second power of the energy scale of new physics $\Lambda$. In the analysis presented here, we have probed the anomalous electroweak top quark using the $t \bar{t}$ production associated with neutrino pair process at the LHC. The 95\% C.L. limits on the Wilson coefficients are computed by focusing on a final state consisting of two opposite-sign charged leptons, missing energy, and two b-tagged jets. A fast simulation of detector effects for an upgraded CMS detector including an average of 200 proton-proton interactions per bunch crossing, is considered. It is found that the $t \bar{t} Z\left(Z \rightarrow \nu_{l} \bar{\nu}_{l}\right)$ production provides the same order sensitivity as $t \bar{t} Z\left(Z \rightarrow l^{+} l^{-}\right)$channel in a HL-LHC scenario with an integrated luminosity of $3 \mathrm{ab}^{-1}$. Better limits are obtained on $c_{\phi t}$ and $c_{\phi Q}^{-}$with respect to the $t \bar{t} Z\left(Z \rightarrow l^{+} l^{-}\right)$channel. The findings indicate that significant statistical power to increase the sensitivity is achieved in the tail of the missing transverse momentum distribution of the $t \bar{t} \nu_{l} \bar{\nu}_{l}$ process.

\section{ACKNOWLEDGMENTS}

M. M. N. would like to thank the CERN Theory Division for the nice hospitality. Authors are grateful to Seyed Mohsen Etesami for the help in the preparation of samples.
[1] S. Weinberg, Phys. Rev. Lett. 43, 1566 (1979).

[2] W. Buchmuller and D. Wyler, Nucl. Phys. B268, 621 (1986).

[3] B. Grzadkowski, M. Iskrzynski, M. Misiak, and J. Rosiek, J. High Energy Phys. 10 (2010) 085.

[4] N. P. Hartland, F. Maltoni, E. R. Nocera, J. Rojo, E. Slade, E. Vryonidou, and C. Zhang, J. High Energy Phys. 04 (2019) 100.

[5] D. de Florian et al. (LHC Higgs Cross Section Working Group), arXiv:1610.07922.

[6] I. Brivio and M. Trott, Phys. Rep. 793, 1 (2019).

[7] A. Falkowski, Pramana 87, 39 (2016).

[8] S. Willenbrock and C. Zhang, Annu. Rev. Nucl. Part. Sci. 64, 83 (2014).

[9] J. A. Aguilar-Saavedra et al., arXiv:1802.07237.

[10] A. Buckley, C. Englert, J. Ferrando, D. J. Miller, L. Moore, M. Russell, and C.D. White, J. High Energy Phys. 04 (2016) 015.

[11] J. Ellis, C. W. Murphy, V. Sanz, and T. You, J. High Energy Phys. 06 (2018) 146.

[12] S. Di Vita, G. Durieux, C. Grojean, J. Gu, Z. Liu, G. Panico, M. Riembau, and T. Vantalon, J. High Energy Phys. 02 (2018) 178 .
[13] A. Falkowski, M. Gonzalez-Alonso, A. Greljo, D. Marzocca, and M. Son, J. High Energy Phys. 02 (2017) 115.

[14] G. Durieux, M. Perelló, M. Vos, and C. Zhang, J. High Energy Phys. 10 (2018) 168.

[15] C. Englert and M. Spannowsky, Phys. Lett. B 740, 8 (2015).

[16] C. Englert, R. Kogler, H. Schulz, and M. Spannowsky, Eur. Phys. J. C 76, 393 (2016).

[17] F. F. Freitas, C. K. Khosa, and V. Sanz, Phys. Rev. D 100, 035040 (2019).

[18] C. Hays, A. Martin, V. Sanz, and J. Setford, J. High Energy Phys. 02 (2019) 123.

[19] F. Maltoni, L. Mantani, and K. Mimasu, J. High Energy Phys. 10 (2019) 004.

[20] F. Demartin, B. Maier, F. Maltoni, K. Mawatari, and M. Zaro, Eur. Phys. J. C 77, 34 (2017).

[21] O. B. Bylund, F. Maltoni, I. Tsinikos, E. Vryonidou, and C. Zhang, J. High Energy Phys. 05 (2016) 052.

[22] M. Schulze and Y. Soreq, Eur. Phys. J. C 76, 466 (2016).

[23] R. Rontsch and M. Schulze, J. High Energy Phys. 08 (2015) 044.

[24] M. Malekhosseini, M. Ghominejad, H. Khanpour, and M. M. Najafabadi, Phys. Rev. D 98, 095001 (2018). 
[25] J. A. Aguilar-Saavedra, C. Degrande, and S. Khatibi, Phys. Lett. B 769, 498 (2017).

[26] S. M. Etesami, S. Khatibi, and M. M. Najafabadi, Eur. Phys. J. C 76, 533 (2016).

[27] C. Englert, P. Galler, and C. D. White, arXiv:1908.05588.

[28] S. M. Etesami and E. D. Roknabadi, Phys. Rev. D 100, 015023 (2019).

[29] R. Jafari, P. Eslami, M. M. Najafabadi, and H. Khanpour, arXiv:1909.00592.

[30] S. M. Etesami, S. Khatibi, and M. M. Najafabadi, Phys. Rev. D 97, 075023 (2018).

[31] M. Koksal, A. A. Billur, A. Gutierrez-Rodriguez, and M. A. Hernandez-Ruiz, arXiv:1905.02564.

[32] K. Y. Oyulmaz, A. Senol, H. Denizli, and O. Cakir, Phys. Rev. D 99, 115023 (2019).

[33] F. Deliot, R. Faria, M. C. N. Fiolhais, P. Lagarelhos, A. Onofre, C. M. Pease, and A. Vasconcelos, Phys. Rev. D 97, 013007 (2018).

[34] E. Boos and V. Bunichev, Phys. Rev. D 101, 055012 (2020).

[35] J. Ellis, V. Sanz, and T. You, J. High Energy Phys. 03 (2015) 157.

[36] S. Khatibi and M. M. Najafabadi, Phys. Rev. D 90, 074014 (2014).

[37] A. Ferroglia, M. C. N. Fiolhais, E. Gouveia, and A. Onofre, Phys. Rev. D 100, 075034 (2019).

[38] C. Englert, K. Nordström, K. Sakurai, and M. Spannowsky, Phys. Rev. D 95, 015018 (2017).

[39] C. Englert and M. Russell, Eur. Phys. J. C 77, 535 (2017).

[40] C. Englert, P. Galler, and C. D. White, Phys. Rev. D 101, 035035 (2020).

[41] I. Brivio, S. Bruggisser, F. Maltoni, R. Moutafis, T. Plehn, E. Vryonidou, S. Westhoff, and C. Zhang, J. High Energy Phys. 02 (2020) 131.

[42] G. Durieux et al., arXiv:1906.12310.

[43] A. Helset, A. Martin, and M. Trott, arXiv:2001.01453.

[44] A. Helset and M. Trott, Phys. Lett. B 795, 606 (2019).

[45] A. Barzinji, M. Trott, and A. Vasudevan, Phys. Rev. D 98, 116005 (2018).

[46] H. Khanpour, arXiv:1909.03998.

[47] R. Contino, A. Falkowski, F. Goertz, C. Grojean, and F. Riva, J. High Energy Phys. 07 (2016) 144.

[48] S. Chatrchyan et al. (CMS Collaboration), J. Instrum. 3, S08004 (2008).

[49] CMS Collaboration, arXiv:1907.11270.

[50] M. Aaboud et al. (ATLAS Collaboration), Phys. Rev. D 99, 072009 (2019).
[51] CMS Collaboration, Report No. CMS-PAS-FTR-18-036.

[52] A. M. Sirunyan et al. (CMS Collaboration), J. High Energy Phys. 02 (2019) 149.

[53] V. Khachatryan et al. (CMS Collaboration), Phys. Lett. B 762, 512 (2016).

[54] J. A. Aguilar-Saavedra, Nucl. Phys. B812, 181 (2009).

[55] R. Röntsch and M. Schulze, J. High Energy Phys. 07 (2014) 091; 09 (2015) 132(E).

[56] J. Bernabeu, D. Comelli, L. Lavoura, and J. P. Silva, Phys. Rev. D 53, 5222 (1996).

[57] W. Hollik, J. I. Illana, S. Rigolin, C. Schappacher, and D. Stockinger, Nucl. Phys. B551, 3 (1999); B557, 407(E) (1999).

[58] A. Czarnecki and B. Krause, Acta Phys. Polon. B 28, 829 (1997), https://www.actaphys.uj.edu.pl/fulltext?series= Reg\&vol $=28 \&$ page $=829$.

[59] J. Alwall, R. Frederix, S. Frixione, V. Hirschi, F. Maltoni, O. Mattelaer, H.-S. Shao, T. Stelzer, P. Torrielli, and M. Zaro, J. High Energy Phys. 07 (2014) 079.

[60] J. Alwall, M. Herquet, F. Maltoni, O. Mattelaer, and T. Stelzer, J. High Energy Phys. 06 (2011) 128.

[61] C. Degrande, C. Duhr, B. Fuks, D. Grellscheid, O. Mattelaer, and T. Reiter, Comput. Phys. Commun. 183, 1201 (2012).

[62] T. Sjostrand, L. Lonnblad, S. Mrenna, and P. Z. Skands, arXiv:hep-ph/0308153.

[63] T. Sjostrand, S. Mrenna, and P. Z. Skands, Comput. Phys. Commun. 178, 852 (2008).

[64] R. D. Ball et al., Nucl. Phys. B867, 244 (2013).

[65] J. de Favereau, C. Delaere, P. Demin, A. Giammanco, V. Lemaître, A. Mertens, and M. Selvaggi (DELPHES 3 Collaboration), J. High Energy Phys. 02 (2014) 057.

[66] D. Contardo, M. Klute, J. Mans, L. Silvestris, and J. Butler, Report Nos. CERN-LHCC-2015-010, LHCC-P-008, CMSTDR-15-02.

[67] M. Cacciari, G. P. Salam, and G. Soyez, Eur. Phys. J. C 72, 1896 (2012).

[68] M. Cacciari, G. P. Salam, and G. Soyez, J. High Energy Phys. 04 (2008) 063.

[69] S. Chatrchyan et al. (CMS Collaboration), J. Instrum. 8, P04013 (2013).

[70] V. Khachatryan et al. (CMS Collaboration), J. Instrum. 12, P01020 (2017).

[71] C. Zhang, Chin. Phys. C 42, 023104 (2018).

[72] C. Zhang, N. Greiner, and S. Willenbrock, Phys. Rev. D 86, 014024 (2012). 